University of Nebraska - Lincoln

DigitalCommons@University of Nebraska - Lincoln

Norman R. Simon Papers

Research Papers in Physics and Astronomy

3-1-1971

Second-Order Pulsations In Polytropes

Norman R. Simon

University of Nebraska - Lincoln, nsimon@unl.edu

Follow this and additional works at: https://digitalcommons.unl.edu/physicssimon

Simon, Norman R., "Second-Order Pulsations In Polytropes" (1971). Norman R. Simon Papers. 10.

https://digitalcommons.unl.edu/physicssimon/10

This Article is brought to you for free and open access by the Research Papers in Physics and Astronomy at DigitalCommons@University of Nebraska - Lincoln. It has been accepted for inclusion in Norman R. Simon Papers by an authorized administrator of DigitalCommons@University of Nebraska - Lincoln. 
The Astrophysical Journal, 164:331-340, 1971 March 1

(C) 1971. The University of Chicago All rights reserved Printed in U S A

\title{
SECOND-ORDER PULSATIONS IN POLYTROPES
}

\author{
NORMAN R. SIMON \\ Institute for Space Studies, Goddard Space Flight Center, NASA I I ew York, New York, and \\ Behlen Laboratory of Physics, * University of Nebraska, Lincoln \\ Received 1970 June 4; revised 1970 October 7
}

\section{ABSTRACT}

A theory due to Eddington is employed to calculate second-order corrections to the usual linear, quasi-adiabatic pulsational amplitudes. Such corrections are necessary in order to evaluate the pulsational stability of stars in thermal imbalance $\left(d S_{0} / d t \neq 0\right)$. The second-order quantities are calculated, and their properties discussed, for a wide variety of polytropic models. Subsequently, a number of these models are tested for pulsational stability, and the conclusions drawn in an earlier, more preliminary work are re-evaluated on the basis of the results. In particular, it is found that stars in pre-main-sequence contraction are probably stable against pulsations energized via thermal imbalance.

\section{INTRODUCTION}

In the linear, quasi-adiabatic theory of stellar pulsations, the pulsational stability of a system is evaluated by means of an integral whose terms are of second order. For convenience, these terms may be divided into two categories: (1) terms independent of the secular rate of change of the equilibrium entropy

$$
\frac{d S_{0}}{d t}=\frac{1}{T}\left(\epsilon-\frac{\partial L_{r}}{\partial m}\right)
$$

and (2) terms proportional to $d S_{0} / d t$.

Following an earlier paper (Simon 1970, hereafter called Paper I), we shall write the stability integral, in the absence of sources or sinks of subatomic energy (i.e., for $\epsilon=0$ ), in the form

$$
\begin{aligned}
\frac{L_{P}}{L}= & -\int_{0}^{1}\left(\Gamma_{3}-1\right) \rho_{*} L^{-1} \frac{\partial}{\partial q}\left(\delta L_{r}\right) d q+\int_{0}^{1}\left(\Gamma_{3}-1\right)^{2} \frac{\partial f}{\partial q}\left(\rho_{*}\right)^{2} d q \\
& -\int_{0}^{1} \frac{\rho^{2}}{2 T} \frac{\partial^{3} U}{\partial S \partial \rho^{2}} \frac{\partial f}{\partial q}\left(\rho_{*}\right)^{2} d q-2 \int_{0}^{1}\left(\Gamma_{3}-1\right) \frac{\partial f}{\partial q}\left\langle\frac{\delta \rho}{\rho}\right\rangle d q,
\end{aligned}
$$

where $\rho_{*}$ indicates the relative first-order (harmonic) perturbation of the equilibrium density, and $\langle\delta \rho / \rho\rangle$ a time average (over the pulsation period) of the total density perturbation, including terms up to second order. (Exactly what we mean here by first- and second-order terms, and how they are obtained, will be discussed in detail in the next section.) In addition, we have defined $q=m / M, f=L_{r} / L$, while the rest of the notation conforms to that of Paper I.

For further convenience we rewrite equation (1) in a shorthand form:

$$
L_{P} / L=I_{1}+I_{2}+J_{2}+K_{2},
$$

where the integrals, including signs, are labeled in order.

The term $I_{1}$ ("ordinary" term) is independent of $d S_{0} / d t$, and is the term usually treated in the linear, quasi-adiabatic analysis. On the other hand, the terms with sub-

* Present address. 
script 2 ("thermal-imbalance" terms) vanish during epochs of equilibrium nuclear burning in stars (i.e., when $d S_{0} / d t \approx 0$ ), and, hence, have generally been ignored.

In Paper I, $I_{2}$ and $J_{2}$ were calculated for a few simple stellar interiors. It was shown there that, for luminosity increasing outward, these terms nearly always tend to energize pulsations, but are small compared with the "ordinary" damping $I_{1}$. For luminosity decreasing outward, $I_{2}$ and $J_{2}$ produce damping. In the final section of Paper I, the suggestion was made that $I_{2}$ and $J_{2}$ might approach or exceed $I_{1}$ in two specific casesnamely, thermally unstable shell-burning stars, and stars in pre-main-sequence contraction.

In the event that contributions due to thermal imbalance do become important, it is obvious that the term $K_{2}$ must also be studied in detail. This requires a second-order theory. In what follows, we propose to calculate second-order pulsational amplitudes according to a simple method originally due to Eddington. We shall apply this method to a wide range of polytropic models, calculating the second-order quantities, using them to evaluate $K_{2}$, and, where possible, comparing the latter in size with the other terms of equation (2). In the final section of this work, some conclusions drawn in Paper I will be reevaluated in light of the second-order calculations.

\section{THE SECOND-ORDER THEORY}

The method we shall use to calculate second-order quantities is given in Rosseland (1949).

We begin with the adiabatic equation of motion

$$
\frac{d^{2} r}{d t^{2}}=-\frac{r^{2}}{\rho_{0} a^{2}} \frac{\partial}{\partial a}\left[P_{0}\left(\frac{a^{2}}{r^{2} r^{\prime}}\right)^{\gamma}\right]-g_{0}\left(\frac{a}{r}\right)^{2}
$$

where the subscript zero indicates equilibrium quantities; $a=r_{0} ; g_{0}=G m / a^{2}$; and the prime denotes differentiation with respect to $a$.

Using

$$
r=a(1+\eta)
$$

in equation (3), and neglecting all powers of $\eta$ and its derivatives higher than the second, we obtain (for $d a / d t=d^{2} a / d t^{2}=0$, and $\gamma=$ const.)

$$
\rho_{0} a \frac{d^{2} \eta}{d t^{2}}(1-2 \eta)=L(\eta)-Q(\eta)
$$

where $L(\eta)$ contains the first-order terms and $Q(\eta)$ the second-order terms. Both functions are given in Rosseland (1949, chapter 7).

The equation for the first-order radius amplitude is just

and has the harmonic solution

$$
L(\eta)=\rho_{0} a \frac{d^{2} \eta}{d t^{2}}
$$

$$
\eta=x_{*} \cos \sigma t \text {. }
$$

We now seek a solution to the second-order equation (5) of the form

$$
\eta=x_{*} \cos \sigma t+w_{*} \cos 2 \sigma t+u_{*},
$$

where $w_{*}$ and $u_{*}$ are of second order.

Inserting equation (6) into equation (5), again neglecting terms higher than second order, and equating coefficients of linearly independent Fourier terms, we finally obtain (Rosseland 1949)

$$
\begin{gathered}
4 \rho_{0} a \sigma^{2} w_{*}+L\left(w_{*}\right)=\rho_{0} a \sigma^{2} x_{*}^{2}+\frac{1}{2} Q\left(x_{*}\right), \\
L\left(u_{*}\right)=\rho_{0} a \sigma^{2} x_{*}^{2}+\frac{1}{2} Q\left(x_{*}\right) .
\end{gathered}
$$


The amplitude $w_{*}$ represents the anharmonic correction to the pulsation introduced by the second-order terms. It was studied by Eddington (1919) and Kluyver (1935), who treated equation (7) for the standard model in attempts to reproduce the radial-velocity curves of Cepheid variables.

The quantity $u_{*}$, on the other hand, gives the average displacement of the center of pulsation over a period. Since it is this term that contributes to the integral $K_{2}$, we shall be interested here in equation (8).

To see how this works out, let us write the equation of continuity in the form

$$
\rho=\frac{\rho_{0} a^{2}}{r^{2} r^{\prime}} .
$$

Inserting equation (4), we obtain to second order

$$
\frac{\rho-\rho_{0}}{\rho_{0}}=\frac{\delta \rho}{\rho}=-\left(3 \eta+a \eta^{\prime}\right)+6 \eta^{2}+a^{2} \eta^{2}+4 a \eta \eta^{\prime}
$$

and using equation (6),

$$
\begin{aligned}
\frac{\delta \rho}{\rho}= & -\left(3 x_{*}+a x^{\prime}\right) \cos \sigma t+\left[3 x_{*}{ }^{2}+\frac{1}{2} a^{2}\left(x^{\prime}\right)^{2}+2 a x_{*} x^{\prime}{ }_{*}-3 w_{*}-a w_{*}^{\prime}\right] \cos 2 \sigma t \\
& +\left[3 x_{*}{ }^{2}+\frac{1}{2} a^{2}\left(x^{\prime}\right)^{2}+2 a x_{*} x^{\prime}{ }_{*}-3 u_{*}-a u_{*}^{\prime}\right],
\end{aligned}
$$

where the coefficient of $\cos \sigma t$ is just the first-order density perturbation

$$
\rho_{*}=-\left(3 x_{*}+a x^{\prime}\right) \text {. }
$$

We then have for the total time-averaged density perturbation

$$
\left\langle\frac{\delta \rho}{\rho}\right\rangle=3 x_{*}{ }^{2}+\frac{1}{2} a^{2}\left(x^{\prime}\right)^{2}+2 a x_{*} x^{\prime}{ }_{*}-3 u_{*}-a u^{\prime},
$$

where the terms involving $\cos \sigma t$ and $\cos 2 \sigma t$ vanish in the integration over the period $=2 \pi / \sigma$.

The integral $K_{2}$ then becomes

$$
K_{2}=-2 \int_{0}^{1}\left(\Gamma_{3}-1\right) \frac{\partial f}{\partial q}\left[3 x_{*}{ }^{2}+\frac{1}{2} a^{2}\left(x^{\prime}\right)^{2}+2 a x_{*} x^{\prime}{ }_{*}-3 u_{*}-a u_{*}^{\prime}\right] d q .
$$

III. THE SECOND-ORDER DISPLACEMENTS

We begin this section by writing out equation (8) in terms of the Emden variables, $x$ and $y$ (British Association for the Advancement of Science 1932):

$$
\begin{gathered}
u^{\prime \prime}{ }_{*}+\left[\frac{(n+1) y^{\prime}}{y}+\frac{4}{x}\right] u_{*}^{\prime}+\frac{(n+1)(3 \gamma-4) y^{\prime}}{\gamma x y} u_{*} \\
=-\frac{(n+1)}{x y}\left[\frac{\omega^{2} x y_{\text {sur }}^{\prime}}{\gamma x_{\text {sur }}} x_{*}{ }^{2}-\frac{[1.5 \gamma(3 \gamma+1)-10] y^{\prime}}{2 \gamma} x_{*}{ }^{2}\right. \\
\left.-\frac{(3 \gamma+1) x y^{\prime}}{2} x_{*} x^{\prime}{ }_{*}-\frac{(\gamma+1) x^{2} y^{\prime}}{4}\left(x^{\prime}\right)^{2}\right] \\
+\frac{2(3 \gamma+1)}{x} x_{*} x^{\prime}+(2 \gamma+1)\left(x^{\prime}{ }^{2}\right)^{2}+\frac{1}{2} x^{\prime \prime}{ }_{*}\left[(3 \gamma+1) x_{*}+(\gamma+1) x x_{*}^{\prime}\right] .
\end{gathered}
$$


Here, $n$ is the polytropic index, $\omega^{2}=\sigma^{2} R^{3} / G M$ the usual dimensionless angular frequency, the subscript "sur" indicates the stellar surface, and the primes denote differentiation with respect to the radial-extension variable $x$.

Equation (10) turns out to be a familiar type of stellar equation, whose solution corresponds, in this case, to a unique starting value $\left.u_{*}\right|_{\text {sur }}$ at the surface of the model. Once the first-order solution is known (i.e., $\omega^{2}, x_{*}$, and its derivatives), trial integrations may be made in the usual way from center out and from surface in (see, e.g., Ezer and Cameron 1963) until a fit is obtained. To begin these integrations, we require starting expansions at both surface and center.

The fitting variables we used were the most obvious ones, $u_{*}$ and $u_{*}^{\prime}$, and these turned out to be also the most convenient. In this manner, equation (10) was integrated for a wide variety of models with different indices $n$ and various first-order modes, including a number of overtones. Strictly speaking, equation (10) is valid for an ideal gas, but not for a gas in which radiation pressure is taken into account. However, it can easily be shown that the error incurred by applying this equation while including radiation pressure is of the order of the ratio

$$
\left.\frac{\partial \ln \Gamma_{1}}{\partial \ln \rho}\right|_{S} /\left(\Gamma_{1}-1\right),
$$

a quantity which is less than 4 percent over the whole range $\frac{4}{3} \leq \Gamma_{1} \leq \frac{5}{3}$. With this in mind, we have calculated a number of $(n=3)$ polytropes with radiation pressure included.

Results of the calculations are given for selected models in Table 1, where, for the ratio $\beta$ of gas pressure to total pressure and for the harmonic overtone under consideration, the rows represent in descending order the dimensionless angular frequency of the first-order (harmonic) mode, the surface and central values of $u_{*}$, the central value of $x_{*}$, the time-averaged total density perturbation (9), and the square of the first-order density perturbation $\boldsymbol{\rho}_{*}$. Since all terms in equation (10) will scale as the square of the first-order amplitude $x_{*}$, all of the pulsational amplitudes in Table 1 have been normalized by the choice $\left.x_{*}\right|_{\text {sur }}=1.0$.

One sees immediately from this table that the second-order corrections $u_{*}$ are always positive at the surface of the model. Furthermore, the values of $\left.u_{*}\right|_{\text {sur }}$ increase monotonically with increasing $\omega^{2}$, without specific reference to any of the physical properties which determine $\omega^{2}$, such as the polytropic index, the amount of radiation pressure, or the overtone being considered. This quality is illustrated in Figure 1 in a plot of $\left.u_{*}\right|_{\text {sur }}$ versus $\omega^{2}$ for most of the models given in Table 1 . The relationship that emerges is very nearly linear and is presented here for possible future use in guessing the starting values $\left.u_{*}\right|_{\text {sur }}$ for models more complex than the polytropes we have treated.

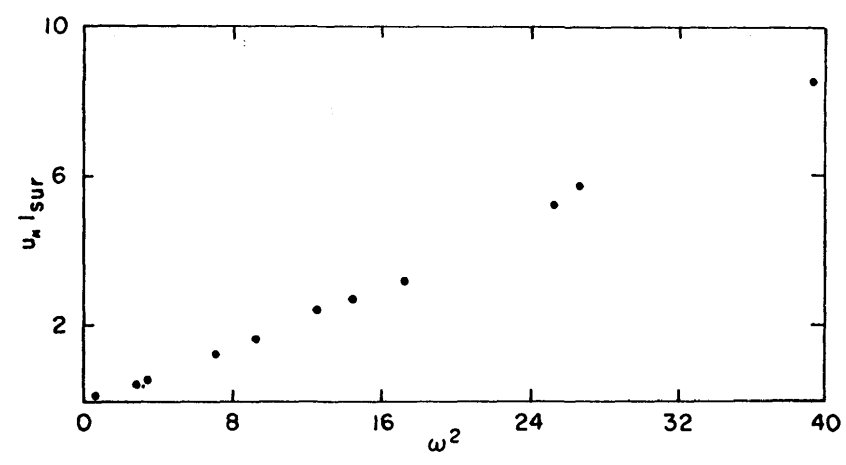

Fig, 1.-Displacement at the surface $\left.u_{*}\right|_{\text {sur }}$ versus $\omega^{2}$ for models of Table 1 
TABLE 1

Pulsational Properties of Selected Models*

\begin{tabular}{|c|c|c|c|c|c|c|c|c|c|c|c|}
\hline \multirow[b]{2}{*}{ VARIABLE } & \multicolumn{3}{|c|}{$n=1.5$} & \multicolumn{7}{|c|}{$n=3.0$} & \multirow{2}{*}{$\begin{array}{c}n=4.5 \\
\begin{array}{c}\beta=1.0 \\
0 \dagger\end{array}\end{array}$} \\
\hline & $\begin{array}{c}\beta=1.0 \\
0 \dagger\end{array}$ & $\begin{array}{c}\beta=1.0 \\
1 \dagger\end{array}$ & $\begin{array}{c}\beta=1.0 \\
2 \dagger\end{array}$ & $\begin{array}{c}\beta=0.999 \\
0 \dagger\end{array}$ & $\begin{array}{c}\beta=0.900 \\
0 \dagger\end{array}$ & $\begin{array}{c}\beta=0.900 \\
1 \dagger\end{array}$ & $\begin{array}{c}\beta=0.900 \\
2 \dagger\end{array}$ & $\begin{array}{c}\beta=0.900 \\
3 \dagger\end{array}$ & $\begin{array}{c}\beta=0.500 \\
0 \dagger\end{array}$ & $\begin{array}{c}\beta=0.100 \\
0 \dagger\end{array}$ & \\
\hline$\omega^{2} \ldots \ldots \ldots$ & 2.71 & 12.5 & 26.6 & 9.22 & 7.08 & 14.4 & 25.2 & 39.3 & 3.33 & 0.663 & 17.2 \\
\hline$\left.u_{*}\right|_{\text {sur }} \ldots \ldots$ & 0.375 & 2.39 & 5.68 & 1.57 & 1.19 & 2.74 & 5.20 & 8.48 & 0.518 & 0.090 & 3.18 \\
\hline$\left.u_{*}\right|_{\text {cen }} \ldots \ldots$ & 0.033 & 0.022 & 0.018 & -0.000 & -0.000 & 0.000 & 0.000 & 0.000 & 0.001 & 0.001 & 0.000 \\
\hline$\left.x_{*}\right|_{\text {cen }} \ldots \ldots \ldots$ & +0.640 & -0.275 & $+\quad 0.208$ & +0.045 & +0.091 & $-\quad 0.022$ & $+\quad 0.008$ & $-\quad 0.004$ & +0.327 & +0.803 & 0.000 \\
\hline$\langle\delta \rho / \rho\rangle_{\text {sur }} \ldots \ldots$ & +2.83 & -0.153 & -23.6 & +1.83 & +3.70 & -0.007 & -16.6 & -58.0 & +4.48 & +3.42 & -6.02 \\
\hline$\left.\rho_{*}{ }^{2}\right|_{\text {sur }} \ldots \ldots \ldots$ & 16.2 & 98.4 & 337 & 63.1 & 50.2 & 138 & 350 & 768 & 26.4 & 11.9 & 162 \\
\hline
\end{tabular}

* Pulsational quantities normalized to $\left.x_{*}\right|_{\text {sur }}=1.0$.

† Number of the harmonic overtone. 
As one moves in from the surface of the model, the second-order corrections $u_{*}$ fall off in a manner qualitatively similar to that of the first-order amplitudes $x_{*}$, but at a considerably faster rate. Figure 2 shows the runs of $u_{*}$ and $x_{*}$ in the model $n=1.5$ for both the fundamental mode and the first overtone. The curves for this polytrope are typical of all those calculated, with the drop-off rates of both $u_{*}$ and $x_{*}$ showing the familiar increase with increasing central condensation of the models (i.e., with increasing $n$ ). It may be noticed from Figure 2 that the correction $u_{*}$ to the first overtone (curve $2 a$ ) has two nodes-exactly double that of the first-order amplitude $x_{*}$ (curve $1 a$ ). This doubling of the number of nodes turned out to be a general characteristic in the models treated, except that in some cases an additional node appeared toward the center in a region of extremely small amplitude. This is illustrated in Table 1 , where one notices, for example, that $\left.u_{*}\right|_{\text {cen }}<0$ for the fundamental mode of the model $n=3, \beta=0.9$. In order to ascertain whether that particular node was real, we tried to make it disappear by means of numerical techniques such as improving the eigenvalues, changing the integration step-size, etc. Since the node always remained, we conclude that it is probably real. In any case, the corrections $u_{*}$ are so small in the regions in question that the pulsation can hardly be affected either way.

We will close this section by pointing out some interesting additional information that can be obtained from Table 1. The second-order theory we have used is an extremely simple one, unable to treat either amplitude-dependent effects or the coupling of pulsational modes. However, since in the derivation of equation (5), terms of the order $x_{*}{ }^{3}$ and $u_{*} x_{*}$ were ignored compared with $x_{*}{ }^{2}$, it follows that $x_{*}$ and $u_{*} / x_{*}$ must be small compared with unity for the theory to be valid. At the same time this is also the criterion for the validity of the linear theory, in which $x_{*}^{2}$ and $u_{*}$ are discarded in comparison with $x_{*}$.

Suppose we scale the entries in Table 1 to fit a first-order surface amplitude $x_{*}=0.1$,

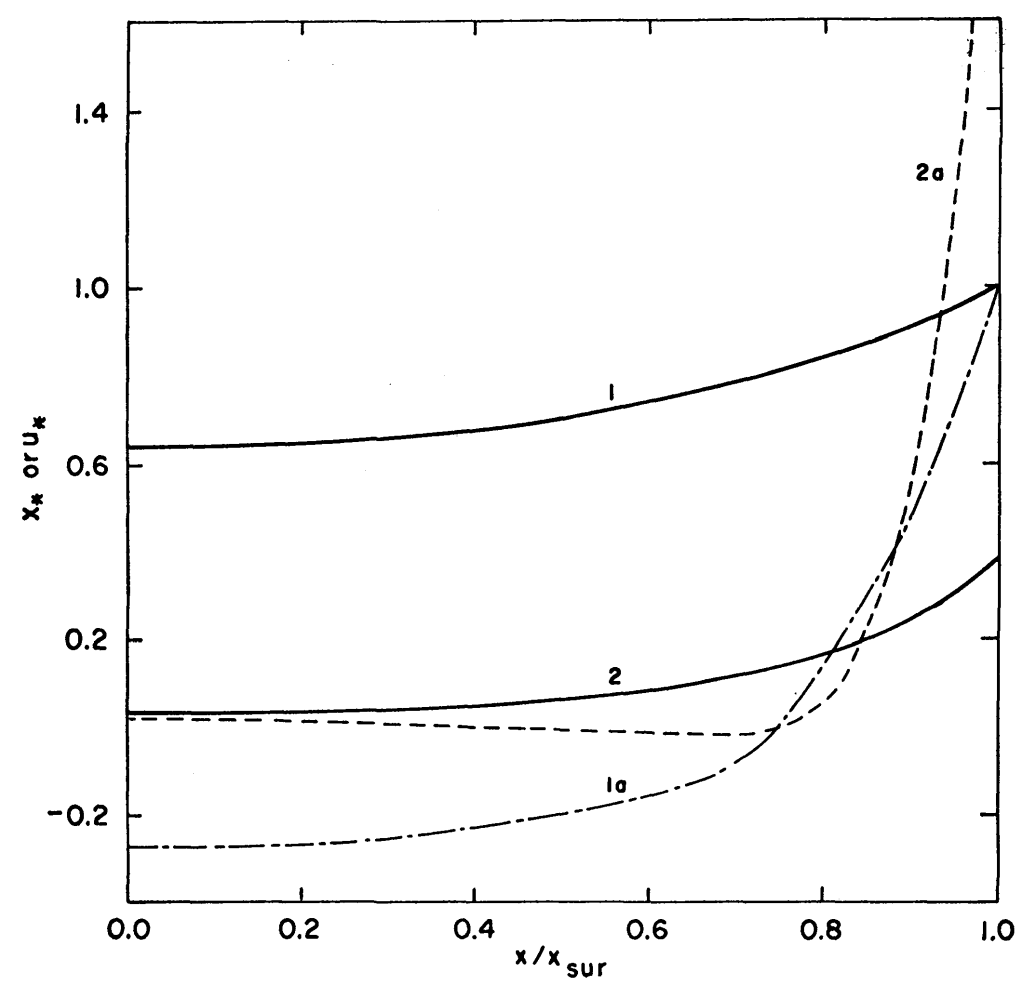

FIG. 2.-Runs of $u_{*}$ and $x_{*}$ for the polytrope $n=1.5$. Fundamental mode: $x_{*}$ (curve 1$), u_{*}($ curve 2). First overtone: $x_{*}$ (curve 1a), $u_{*}$ (curve 2a). Abscissa represents the normalized radius. 
and consider as an example the fundamental and first two overtones of the model $n=3$, $\beta=0.9$. Then one sees from the table that the surface ratios $\left(u_{*} / x_{*}\right)_{\text {sur }}$ for these cases are, respectively, $0.12,0.27$, and 0.52 . It can be concluded from this that, for a first-order surface amplitude of 10 percent, the linear theory suffices for the fundamental and perhaps the first overtone, but certainly not for the second overtone. Since $\left(u_{*} / x_{*}\right)_{\text {sur }}$ is a monotonically increasing function of $\omega^{2}$ (Fig. 1), it follows from this example that the larger $\omega^{2}$, the smaller the amplitude at which the linear analysis fails and higher-order effects must be taken into account. Thus we see that the second-order theory of Eddington, unsuitable for treating amplitude dependence is nonetheless able to provide a rough upper bound on the region where the linear theory will be valid.

\section{THE STABILITY INTEGRAL}

\section{a) The Density Perturbation}

The total time-averaged density perturbation $\langle\delta \rho / \rho\rangle$ is given by equation (9). One can see that it contains two kinds of terms - namely, those involving the second-order corrections $u_{*}$, and those involving $x_{*}{ }^{2}$. As Figure 2 illustrates, the quantities $u_{*}$ and $u_{*}^{\prime}$ are both positive in the large-amplitude regions that determine the stability integrals. This corresponds to the fact that, on the average over a period, the center of pulsation of a given particle is displaced toward longer radii (see eq. [6]), a circumstance which tends to reduce the average density. On the other hand, the terms in equation (9) which involve $x_{*}^{2}$ tend to increase the density, and it is this competition which finally determines the sign of $\langle\delta \rho / \rho\rangle$.

Table 1 lists the surface values of $\langle\delta \rho / \rho\rangle$ for the calculated models. One notices the general trend from positive to negative values with increasing $\omega^{2}$, reflecting the increasing size of $u_{*}$ compared with $x_{*}^{2}$ as $\omega^{2}$ gets larger. Also listed for comparison are the squares of the first-order density perturbations $\left.\rho_{*}{ }^{2}\right|_{\text {sur. }}$.

Let us return for a moment to equation (1). Suppose that the luminosity is increasing outward $(\partial f / \partial q>0)$, and consider the sign of the last integral, i.e., $K_{2}$. This sign will be opposite to the preponderant sign of $\langle\delta \rho / \rho\rangle$ over the integration. Let us state here that, for all the cases calculated, $\langle\delta \rho / \rho\rangle$ was always preponderantly positive (even when its surface value was negative), and thus $K_{2}$ was always negative (i.e., a damping term) for luminosity increasing outward. For luminosity decreasing outward, $K_{2}$ would of course produce energizing.

\section{b) The Polytrope $n=3$}

Actual evaluation of the stability integral requires that the luminosity distribution $f(q)$ be known. For the standard model $(n=3)$, we have chosen the distribution $f=q$ as a physically reasonable case (Paper I; Deinzer and Hansen 1969). Table 2 gives the various terms in the stability integral for a number of models selected from Table 1. The

TABLE 2

VALUES OF THE STABILITY INTEGRALS

\begin{tabular}{|c|c|c|c|c|c|c|}
\hline \multirow[b]{2}{*}{ VARIABLE } & \multicolumn{2}{|c|}{$n=1.5$} & \multicolumn{4}{|c|}{$n=3$} \\
\hline & $\beta=1.0$ & $\beta=1.0$ & $\beta=0.999$ & $\beta=0.900$ & $\beta=0.500$ & $\beta=0.100$ \\
\hline $\begin{array}{l}\omega^{2} \ldots \ldots \ldots \\
I_{1} \ldots \ldots \ldots \ldots \\
I_{2} \ldots \ldots \ldots \ldots \ldots \\
J_{2} \ldots \ldots \ldots \ldots \ldots \\
K_{2} \ldots \ldots \ldots \ldots \ldots \\
L_{P} / L \ldots \ldots \ldots \ldots\end{array}$ & $\begin{array}{r}2.71 \\
-12.6 \\
2.25 \\
0.56 \\
-1.88 \\
-11.7\end{array}$ & $\begin{aligned} & 12.5 \\
&-84.5 \\
& 0.28 \\
& 0.07 \\
&-0.25 \\
&-84.4\end{aligned}$ & $\begin{array}{rl} & 9.22 \\
-51.7 & 0.07 \\
0.02 \\
-0.05 \\
-51.6\end{array}$ & $\begin{array}{r}7.08 \\
-22.2 \\
0.08 \\
0.05 \\
-0.08 \\
-22.1\end{array}$ & $\begin{array}{r}3.33 \\
-4.57 \\
0.21 \\
0.19 \\
-0.36 \\
-4.53\end{array}$ & $\begin{array}{r}0.663 \\
-0.68 \\
0.72 \\
0.70 \\
-1.40 \\
-0.66\end{array}$ \\
\hline
\end{tabular}


$n=3$ models differ in radiation pressure, encompassing the wide range $0.999 \leq \beta \leq 0.1$.

Since the terms $I_{1}, I_{2}$, and $J_{2}$ were discussed in detail in Paper I, our interest here shall be in the final term $K_{2}$. As indicated above, $K_{2}$ is a damping term. It has a magnitude approximately equal to those of the energizing terms $I_{2}$ and $J_{2}$ and thus tends to offset them. The "thermal-imbalance" terms are always much smaller than the "ordinary" damping $I_{1}$, except in the case of overwhelming radiation pressure $(\beta=0.1)$, where $K_{2}$ actually gives the largest contribution and $I_{1}$ the smallest. Even in this case, however, the pulsation is damped, though by a relatively small margin.

\section{c) The Polytrope $n=1.5$}

We choose here the luminosity distribution corresponding to a star in homologous contraction. In that case, with $\gamma=\frac{5}{3}$, we have, in Emden variables,

$$
\frac{\partial f}{\partial x}=\frac{7}{5 x_{\text {sur }}^{3}\left(y_{\text {sur }}^{\prime}\right)^{2}} x^{2} y^{2.5}
$$

Table 2 lists the terms in the stability integral for the fundamental mode and the first overtone. Once more, the "ordinary" damping $I_{1}$, calculated here as if the star were wholly radiative, completely dominates, whereas the terms due to thermal imbalance tend to cancel.

It is well known that the model discussed here is a reasonable approximation to the fully convective phase of pre-main-sequence contraction. In fact, this polytrope was used by Okamoto (1967) to discuss the pulsational stability of stars in such a phase. With the aid of a method due to Unno (1967), Okamoto recalculated the damping $I_{1}$, taking into account the coupling between convection and radial pulsation. His results, which are mass dependent, show that the damping is considerably weaker, as compared with the radiative case, especially at low masses. Table 3 briefly reproduces these results.

With the damping thus substantially lowered, Okamoto explored the possibility that energizing due to thermal imbalance might be enough to excite pulsations in such models. He evaluated the integral $I_{2}$ in a quite direct manner, and then used an approximate scheme given by Kato and Unno (1967) in order to calculate the sum $\left(J_{2}+K_{2}\right)$. This scheme sought, via approximations, to avoid calculation of the second-order displacements $u_{*}$.

The results of Okamoto (1967) are compared with those of the present work in Table 4. It can be seen that the values of $I_{2}$ agree reasonably well, the difference being due to the rather rough first-order solutions (Lucas 1956) employed by Okamoto. On the other hand, when one compares values of the sum $\left(J_{2}+K_{2}\right)$, it becomes evident that a serious discrepancy exists, with the two results not even agreeing in sign. In view of the straightforward nature of the present work, it would appear that this discrepancy should be attributed to the inadequacy of the above-noted approximation of Kato and Unno (1967); and, indeed, a careful examination of the latter work serves to confirm this. The last line of Table 4 gives the total contribution of the "thermal-imbalance" terms. In both cases, this contribution turns out to be positive (i.e., energizing), but with a significant difference in magnitude.

TABLE 3

“ORDINARY" DAMPING GIVEN BY OKAMOTO (1967) FOR THE POLYTROPE $n=1.5$

\begin{tabular}{ccccc}
\hline \hline$M / M_{\odot}$ & 0.4 & 0.6 & 1.0 & 2.0 \\
\hline$I_{1} \ldots \ldots$ & -1.16 & -1.20 & -1.79 & -6.12 \\
\hline
\end{tabular}


TABLE 4

\section{COMPARISON OF "THERMAL IMBALANCE" TERMS} FOR THE POLYTROPE $n=1.5$

\begin{tabular}{|c|c|c|}
\hline Variable & Okamoto (1967) & Present work \\
\hline $\begin{array}{l}I_{2} \ldots \ldots \ldots \cdots \\
J_{2} \ldots \ldots \cdots \cdots \\
K_{2} \ldots \ldots \cdots \cdots \\
\left(J_{2}+K_{2}\right) \ldots \cdots \cdots \cdots \\
I_{2}+J_{2}+K_{2} \ldots \ldots \ldots \cdots\end{array}$ & $\begin{array}{l}2.15 \\
\ldots \\
1.66 \\
3.81\end{array}$ & $\begin{array}{r}2.25 \\
0.56 \\
-1.88 \\
-1.32 \\
0.93\end{array}$ \\
\hline
\end{tabular}

Thus, when one compares the damping (i.e., $I_{1}$; see Table 3 ) with the energizing (last line of Table 4), it is seen that, if one uses Okamoto's value for the latter, pulsations will be excited in masses less than 1-2 $M_{\odot}$; on the other hand, if one uses the value of the energizing given in the present work, all of the masses in Table 3 are found to be stable. We must conclude that the assertion of Okamoto, that stars with masses less than 1-2 $M_{\odot}$, in pre-main-sequence contraction become pulsationally unstable, cannot be maintained, at least on the basis of the polytropic model.

It should be noted, however, that the margin of stability here is rather small, especially at the lower masses. Coupled with this is the fact that the polytropic representation of pre-main-sequence contraction is certainly oversimplified. In particular, this representation ignores the undoubted existence of ionization zones, whose presence might have an important enhancing effect on the energizing integral $J_{2}$ (Paper I). On the other hand, the pulsation-convection theory of Unno (1967) is rather generous in reducing the "ordinary" damping $I_{1}$ from its radiative value, and, in view of the primitive state of our knowledge of convection, can hardly be considered certain. In summary, it seems fair to say that, although the possibility of energizing pulsations in pre-main-sequence stars via thermal imbalance now appears somewhat improbable, the question nonetheless remains open.

\section{DISCUSSION}

In Paper I, a number of tentative conclusions were drawn regarding the influence of thermal imbalance on pulsational stability. This work was based on a stability integral that was incomplete due to omission of the term $K_{2}$. With that omission rectified in the present investigation, we may now assert the following:

1. For stars crossing the H-R diagram in post-main-sequence evolution and for degenerate stars cooling via photon emission, the "thermal-imbalance" terms are very small compared with "ordinary" damping terms and can thus be ignored.

2. Stars in pre-main-sequence contraction probably do not become pulsationally unstable as suggested by Okamoto (1967), but the question can be settled definitely only by detailed models (including ionization zones), and is subject to the uncertainties inherent in present theories of convection.

3. The pulsational stability of thermally unstable shell-burning stars (Rose 1967, 1968) depends critically on effects due to thermal imbalance. The luminosity distribution above the shell in these stars (luminosity decreasing outward) is such that $I_{2}$ and $J_{2}$ produce damping, while $K_{2}$ is an energizing term.

Finally, we note that all of the above conclusions are, of course, subject to quantitative correction, depending upon the accuracy of the various approximate schemes we have employed. In particular, we emphasize that nonadiabatic effects have not been taken into account, and that the results obtained here can only be as good as the quasiadiabatic approximation allows. 
The author gratefully acknowledges support of an NRC post-doctoral research associateship under the National Aeronautics and Space Administration.

\section{REFERENCES}

British Association for the Advancement of Science 1932, Mathematical Tables, Vol. 2. Deinzer, W., and Hansen, C. J. 1969, Astr. and Ap., 3, 214.

Eddington, A. S. 1919, M.N.R.A.S., 79, 177.

Ezer, D., and Cameron, A. G. W. 1963, Icarus, 1, 422.

Kato, S., and Unno, W. 1967, Pub. Astr. Soc. Japan, 19, 1.

Kluyver, H. A. 1935, B.A.N., 7, 265.

Lucas, D. 1956, Bull. Soc. Roy. Sci. Liège, 25, 585.

Okamoto, I. 1967, Pub. Astr. Soc.Japan, 19, 384.

Rose, W. 1967, Ap.J., 150, 193.

. 1968, ibid., 152, 245.

Rosseland, S. 1949, The Pulsation Theory of Variable Stars (Oxford: Clarendon Press). Simon, N. R. 1970, Ap.J., 159, 859 (Paper I).

Unno, W. 1967, Pub. Astr. Soc. Japan, 19, 140. 